\title{
Real-space simulations of spin-polarized electronic transitions in iron
}

\author{
P. Schattschneider \\ Institut für Festkörperphysik, Technische Universität Wien, A-1040 Wien, Austria \\ J. Verbeeck \\ EMAT, University of Antwerp, Groenenborgerlaan 171, B-2020 Antwerp, Belgium \\ V. Mauchamp and M. Jaouen \\ Institut Pprime, ENSMA, UPR 3346, SP2MI, CNRS-Université de Poitiers, Bd M. et P. Curie, BP 30179, \\ 86962 Futuroscope-Chasseneuil Cedex, France \\ A.-L. Hamon \\ LMSSMAT, École Centrale Paris, Grande Voie des Vignes, F-92290 Chatenay-Malabry, France \\ (Received 29 July 2010; revised manuscript received 7 September 2010; published 8 October 2010)

\begin{abstract}
After the advent of energy-loss magnetic chiral dichroism (EMCD) in 2006, rapid progress in theoretical understanding and in experimental performance was achieved, recently demonstrating a spatial resolution of better than $2 \mathrm{~nm}$. Similar to the x-ray magnetic circular dichroism technique, EMCD is used to study atom specific magnetic moments. The latest generation of electron microscopes opens the road to the mapping of spin moments on the atomic scale with this method. Here the theoretical background to reach this challenging aim is elaborated. Numerical simulations of the $L_{3}$ transition in an Fe specimen, based on a combination of the density-matrix approach for inelastic electron scattering with the propagation of the probe electron in the lattice potential indicate the feasibility of single spin mapping in the electron microscope.
\end{abstract}

DOI: $10.1103 /$ PhysRevB.82.144418

PACS number(s): 78.20.Fm, 71.70.Ej, 82.80.Dx, 71.15.Mb

\section{INTRODUCTION}

Shortly after the discovery of the energy-loss magnetic chiral dichroism (EMCD) effect ${ }^{1}$ impressive improvement in signal and spatial resolution has been achieved. Several laboratories are now beginning to use this technique. ${ }^{2-10}$ There are other methods to map magnetic properties, in particular, x-ray magnetic circular dichroism (XMCD) and electron holography. XMCD is a well-established synchrotron-based technique to probe atom-specific magnetic moments. Using diffractive $\mathrm{x}$-ray optics or lensless imaging ${ }^{11}$ resolutions better than $20 \mathrm{~nm}$ have been reported, ${ }^{12}$ but on a routine basis the achievable spatial limit is of order $50 \mathrm{~nm}$. Electron holography is an order of magnitude better in terms of spatial resolution but is only sensitive to the local magnetic field component perpendicular to the probing beam, i.e., it is not atom specific and needs a Lorentz lens. ${ }^{13}$ EMCD combines the elemental specificity of XMCD with the high spatial resolution of the electron microscope. Although the routine investigation of nanomagnetic systems may appear remote the detection of atom specific magnetic moments on a nanometer scale ${ }^{14}$ bears promise for spintronics applications. Used in combination with sum rules it is even possible to separate spin and orbital moments. ${ }^{15-18}$

The basic mechanism underlying EMCD is the same as for XMCD, the excitation of an electronic transition with change in the magnetic quantum number of an atom by \pm 1 (chiral transition). In view of the intrinsic high lateral resolution of the electron microscope a crucial question related to EMCD is then: is it possible to map chiral transitions on the atomic scale with last generation instruments? If this is so, one would have a unique method at hand to monitor the change in the angular momentum of single electrons during an electronic transition. Spin-orbit coupling would equally allow to map element specific magnetic moments of single atomic columns in the electron microscope. Apart from the interest in understanding the physical mechanism this possibility would constitute a breakthrough in solid-state spectroscopy, opening a distinct way for direct (real-space) study of nanomagnetic materials. The standard EMCD setup uses a parallel beam of incident electrons and measures an energyloss spectrum at a particular point in the diffraction plane..$^{1,19}$ A serious disadvantage of this original setup is the low intensity. Several improvements have been achieved since, increasing the signal strength by almost 2 orders of magnitude. ${ }^{2,20}$ The new generation of electron microscopes with high brightness sources, aberration correctors, and improved energy filters hold promise for detection of single atomic spins via spin-polarized electronic transitions. In a short paper ${ }^{21}$ — hereafter referred to as paper I-it was shown theoretically that magnetic transitions can be made visible in energy-filtered high-resolution (HR) electron microscopic images. That analysis was based on the assumption that a particular transition channel with $\Delta m \neq 0$ can be selected in a single atom suspended in vacuo, i.e., that the $m$ degeneracy of the final state is sufficiently lifted in the magnetic field of the objective lens in order to separate transition channels in the energy loss. This is a very unrealistic case that was chosen to demonstrate the principle. However, a recent numerical result showed that this property holds also for ferromagnetic $\mathrm{Fe}$ where the final states with different magnetic quantum numbers are almost degenerate. ${ }^{22}$ That paper concentrated on a feasibility experiment demonstrating that an EMCD signal could be detected with atomic resolution under optimized experimental conditions. 
Contrary to Ref. 22 we focus here on the underlying theoretical description, combining the propagator for the elastic wave field with transition probabilities calculated within density-functional theory (DFT) in an all-electron scalar relativistic generalized gradient approximation. ${ }^{23}$ Details of the simulation of spin-polarized transitions in a thin crystal of bcc ferromagnetic iron are given. It is shown that spin-orbit coupling in the $3 d$ ferromagnets in combination with the spin polarization of the final state allows the selection of chiral magnetic transition channels with existing energy filters. Bragg diffraction of the probe electron changes the energyfiltered HR image as compared to single-atom mapping by exchange of angular momentum with the lattice, but the images retain their characteristic asymmetry, making it feasible to analyze magnetic transitions on an atomic level with image simulation tools.

We start with the expression for the probability of a chiral transition in conventional transmission electron microscopy (TEM) geometry with the elastic electron propagator before and after inelastic scattering. Contrary to paper I we use an expansion of the mixed dynamic form factor (MDFF) into spherical harmonics, including the relativistic formulation of spin-orbit coupling. The final expression is numerically evaluated for ferromagnetic $\mathrm{Fe}$, simulating the HR images for various thicknesses. The images show a typical asymmetry that can be interpreted in terms of the transferred angular momentum.

\section{MIXED DYNAMIC FORM FACTOR}

After inelastic interaction, the probe electron is in a mixed state, best described by its density matrix. ${ }^{24,25}$ This concept has been proposed for fast electron scattering by Dudarev ${ }^{26}$ and was further developed by Schattschneider et al. ${ }^{27} \mathrm{Using}$ the convention $\mathbf{X}=(\mathbf{x}, z), \mathbf{R}=(\mathbf{r}, z)$, i.e., bold capitals stand for three space vectors and lowercase bold letters for vectors in the lateral plane, the kinetic equation ${ }^{26}$ for the density matrix $\rho_{o}$ of the inelastically scattered electron at positions $\mathbf{R}, \mathbf{R}^{\prime}$ (usually both in the exit plane of the specimen) is in single inelastic scattering approximation

$$
\rho_{o}\left(\mathbf{R}, \mathbf{R}^{\prime}\right)=\int P(\mathbf{R}, \mathbf{X}) P^{*}\left(\mathbf{R}^{\prime}, \mathbf{X}^{\prime}\right) T\left(\mathbf{X}, \mathbf{X}^{\prime}\right) \rho_{i}\left(\mathbf{X}, \mathbf{X}^{\prime}\right) d^{3} X d^{3} X^{\prime},
$$

where the Green's function $P$ propagates the electron in the crystal potential from $\mathbf{X}$ to $\mathbf{R}$, and the inelastic scattering kernel $T$ is the Fourier transform of the MDFF $S$ multiplied by a Lorentzian distribution in momentum representation describing the Coulomb coupling function, and a phase factor that depends on the propagation depth $z, z^{\prime}$ (Ref. 20)

$$
T\left(\mathbf{X}, \mathbf{X}^{\prime}\right)=\mathcal{F}_{\mathbf{q},-\mathbf{q}^{\prime}}\left[S\left(\mathbf{Q}, \mathbf{Q}^{\prime}\right) Q^{-2} Q^{\prime-2}\right] e^{i q_{E}\left(z-z^{\prime}\right)},
$$

where $\mathcal{F}_{\mathbf{a}, \mathbf{b}}$ is the Fourier transform with respect to variables $\mathbf{a}$ and $\mathbf{b}$ and $\mathbf{Q}=\left(\mathbf{q}, q_{E}\right)$ the wave-vector transfer, $\mathbf{q}$ being the two-dimensional coordinate in the diffraction plane, $q_{E}$ $=k E / \gamma m_{0} v^{2}$ the relativistically corrected characteristic scattering angle for incident wave number $k$ and energy-loss $E$, and $Q$ the norm of vector $\mathbf{Q}$.
The density matrix $\rho_{i}\left(\mathbf{X}, \mathbf{X}^{\prime}\right)$ describes the incident electron at positions $\left(\mathbf{X}, \mathbf{X}^{\prime}\right)$ in the specimen. The integral in Eq. (1) is over the whole specimen in three dimensions (3D) and $E$ is implicitly fixed in the expression of $T$ and omitted for convenience.

The single inelastic scattering approximation is valid for core losses in specimens of thickness much smaller than the mean free path [about $100 \mathrm{~nm}$ at typical kinetic energies of the probing electron in TEM (Ref. 28)].

In paper I it was shown that the energy-filtered HR images of electronic transitions with a given change in the magnetic quantum number show a characteristic asymmetry with respect to the center of the atom. As already mentioned in Sec. I, paper I was intended to demonstrate the underlying principle by choosing a rather hypothetical example, namely, the $\mathrm{Si} L$ edge. In realistic cases (such as the $3 d$ ferromagnets) the physics is different: we have degenerate $m$ states, and the dichroic signal rather shows the spin polarization which has been neglected in paper I. Moreover, it is well known that the density matrix of the probe electron, via propagation through the crystal potential, will change as a function of thickness in a nontrivial way. ${ }^{29,30}$ We can therefore not expect to see the clear chiral character of a transition as demonstrated in paper I.

In this section we extend the previous results to degenerate $m$ states in the $3 d$ ferromagnets. It will be shown that spin-orbit coupling of the initial states renders the transfer of angular momentum visible under particular conditions.

It was shown in paper I that in the case of a single atom at axis position $z=z^{\prime}=0$, Eq. (1) simplifies to

$$
\begin{aligned}
\rho_{o}\left(\mathbf{r}, \mathbf{r}^{\prime}\right)= & \left(\frac{2 \pi m e^{2}}{\varepsilon_{0} \hbar^{2} k}\right)^{2} \mathcal{F}_{z}\left[X^{-1}\right]_{q_{E}} \mathcal{F}_{z^{\prime}}\left[X^{\prime-1}\right]_{q_{E}} *_{\mathbf{x}, \mathbf{x}^{\prime}} \\
& \times \int d z d z^{\prime} S\left(\mathbf{X}, \mathbf{X}^{\prime}\right) e^{i q_{E}\left(z-z^{\prime}\right)},
\end{aligned}
$$

where $*_{\mathbf{x}, \mathbf{x}^{\prime}}$ denotes convolution with respect to the lateral (in-plane) coordinates and $\mathbf{r}$ is a vector in the specimen's plane $z$.

$S\left(\mathbf{X}, \mathbf{X}^{\prime}\right)$ is the $3 \mathrm{D}$ Fourier transform of the mixed dynamic form factor so one can rewrite the diagonal element of the density matrix Eq. (4), $I(\mathbf{r}):=\rho(\mathbf{r}, \mathbf{r})$ as

$$
\begin{aligned}
I(\mathbf{r})= & \left(\frac{2 \pi m e^{2}}{\varepsilon_{0} \hbar^{2} k}\right)^{2} \mathcal{F}_{z}\left[X^{-1}\right] \mathcal{F}_{z^{\prime}}\left[X^{\prime-1}\right] *_{\mathbf{x}, \mathbf{x}^{\prime}} \mathcal{F}_{\mathbf{q},-\mathbf{q}^{\prime}} \\
& \times\left.\left[S\left(\mathbf{Q}, \mathbf{Q}^{\prime}\right)\right]\right|_{q_{E}, \mathbf{r}=\mathbf{r}^{\prime}} \cdot
\end{aligned}
$$

Recognizing that ${ }^{27} \mathcal{F}_{z}\left[X^{-1}\right]=\mathcal{F}_{\mathbf{q}}\left[Q^{-2}\right]$,

Equation (4) is, by virtue of the convolution theorem

$$
I(\mathbf{r})=\left.\left(\frac{2 \pi m e^{2}}{\varepsilon_{0} \hbar^{2} k}\right)^{2} \mathcal{F}_{\mathbf{q},-\mathbf{q}^{\prime}}\left[S\left(\mathbf{Q}, \mathbf{Q}^{\prime}\right) Q^{-2} Q^{\prime-2}\right]\right|_{q_{E}, \mathbf{r}=\mathbf{r}^{\prime}} .
$$

Comparison with Eq. (2) shows that

$$
I(\mathbf{r})=\left.\left(\frac{2 \pi m e^{2}}{\varepsilon_{0} \hbar^{2} k}\right)^{2} T(\mathbf{R}, \mathbf{R})\right|_{z=z^{\prime}=0}:=T(\mathbf{r}, \mathbf{r}) .
$$

We give now an expression for the MDFF, including transitions with spin-orbit coupling, and wave functions in a crys- 
tal. The use of Wigner $3 j$ symbols will allow easy collection of terms for a given transfer of angular momentum. In one electron, first-order Born approximation the MDFF is ${ }^{1}$

$$
\begin{aligned}
S\left(\mathbf{Q}, \mathbf{Q}^{\prime}, E\right)= & \sum_{m} \sum_{L M S} \sum_{L^{\prime} M^{\prime} S^{\prime}} \sum_{\lambda \mu} \sum_{\lambda^{\prime} \mu^{\prime}} 4 \pi i^{\lambda-\lambda^{\prime}}(2 l+1) \\
& \times \sqrt{\left[\lambda, \lambda^{\prime}, L, L^{\prime}\right]} Y_{\mu}^{\lambda}(\mathbf{Q} / Q)^{*} Y_{\mu^{\prime}}^{\lambda^{\prime}}\left(\mathbf{Q}^{\prime} / Q^{\prime}\right) \\
& \times\left\langle j_{\lambda}(q)\right\rangle_{E L S j}\left\langle j_{\lambda^{\prime}}\left(q^{\prime}\right)\right\rangle_{E L^{\prime} S^{\prime} j}\left(\begin{array}{lll}
l & \lambda & L \\
0 & 0 & 0
\end{array}\right) \\
& \times\left(\begin{array}{ccc}
l & \lambda^{\prime} & L^{\prime} \\
0 & 0 & 0
\end{array}\right)\left(\begin{array}{ccc}
l & \lambda & L \\
-m & \mu & M
\end{array}\right)\left(\begin{array}{ccc}
l & \lambda^{\prime} & L^{\prime} \\
-m & \mu^{\prime} & M^{\prime}
\end{array}\right) \\
& \times \sum_{j_{z}}(2 j+1)\left(\begin{array}{ccc}
l & \frac{1}{2} & j \\
m & S & -j_{z}
\end{array}\right)\left(\begin{array}{ccc}
l & \frac{1}{2} & j \\
m & S^{\prime} & -j_{z}
\end{array}\right) \\
& \times \sum_{\vec{k} n} D_{L M S}^{\overrightarrow{k n}}\left(D_{L^{\prime} M^{\prime} S^{\prime}}^{\vec{k} n} \delta\left(E+E_{n l j}-E_{\vec{k} n}\right),\right.
\end{aligned}
$$

where $(l, m)$ and $(L, M)$ are, respectively, initial and final angular-momentum quantum numbers, $S$ is the final spin state $( \pm 1 / 2), j, j_{z}$ are the initial state relativistic (spin plus orbit) quantum numbers, $(\lambda, \mu)$ is the multipole index of the Rayleigh expansion $e^{i \mathbf{q} \cdot \mathbf{r}}=\Sigma_{\lambda, \mu} j_{\lambda}(q r) Y_{\lambda}^{\mu}(\mathbf{q} \cdot \mathbf{r} / q r)$, and $D_{L M S}^{\vec{k} n}$ is the projection of the final-state Bloch wave with index $\vec{k} n$ onto an LMS basis. The radial integrals are

$$
\left\langle j_{\lambda}(q)\right\rangle_{E L S j}=\int d r r^{2} u_{L S}^{E}(r) R_{j S}(r) j_{\lambda}(q r)
$$

with the radial parts of the initial and final wave functions $R_{j S}(r), u_{L S}^{E}(r)$, and we use the abbreviation

$$
\left[\lambda, \lambda^{\prime}, l^{\prime}, L^{\prime}\right]:=(2 \lambda+1)\left(2 \lambda^{\prime}+1\right)\left(2 l^{\prime}+1\right)\left(2 L^{\prime}+1\right) .
$$

It is worth mentioning that the expression of the MDFF given by Eq. (7) is only valid when considering inelastic processes that can be approximated by one-particle excitations (i.e., interband transitions between one-electron wave functions). Strictly speaking, this is not the case when considering the $L_{23}$ edge of transition metals where fine structures can be modified by multiplet effects. ${ }^{31}$ However, oneparticle calculations based on a multiple scattering approach proved to give good results in the simulation of $\mathrm{x}$-ray magnetic circular dichroism obtained at the Ni $L_{2,3}$ edge. ${ }^{32}$ This shows that such measurements are only weakly affected by the satellites peaks due to multiplet effects and that the oneparticle approximation is a good approach for the interpretation of such experiments.

Dipole-allowed transitions in the $L_{23}$ edge are then $2 p$ $\rightarrow n d$ and $2 p \rightarrow n s$. Since the latter are an order of magnitude smaller than the former we keep only $2 p \rightarrow n d$. Application of the electric-dipole selection rules restricts the quantum numbers in Eq. (7) to $l=1, \lambda=\lambda^{\prime}=1, L=L^{\prime}=2$. The spinorbit coupling of the final states can be neglected as compared to that of the core state. Consequently, cross terms with $S \neq S^{\prime}$ vanish. In this case, and for symmetries higher than orthorhombic, the cross terms $M \neq M^{\prime}$ in the sums above vanish also ${ }^{33}$ and Eq. (7) simplifies to

$$
\begin{aligned}
S\left(\mathbf{Q}, \mathbf{Q}^{\prime}, E\right)= & 24 \pi \sum_{m} \sum_{M S} \sum_{\mu \mu^{\prime}} Y_{\mu}^{1}(\mathbf{Q} / Q)^{*} Y_{\mu^{\prime}}^{1}\left(\mathbf{Q}^{\prime} / Q^{\prime}\right) \\
& \times\left\langle j_{1}(q)\right\rangle_{E 2 S j}\left\langle j_{1}\left(q^{\prime}\right)\right\rangle_{E 2 S j}\left(\begin{array}{ccc}
1 & 1 & 2 \\
-m & \mu & M
\end{array}\right) \\
& \times\left(\begin{array}{ccc}
1 & 1 & 2 \\
-m & \mu^{\prime} & M
\end{array}\right) \sum_{j_{z}}(2 j+1) \\
& \times\left(\begin{array}{ccc}
1 & \frac{1}{2} & j \\
m & S & -j_{z}
\end{array}\right) \sum_{\vec{k} n} D_{2 M S}\left(D_{2 M S}^{\vec{k} n}\right)^{*} \\
& \times \delta\left(E+E_{n l j}-E_{\vec{k} n}\right) .
\end{aligned}
$$

The first two $3 j$ symbols force $\mu^{\prime}=\mu$. Assuming that the final density of states (DOS) does not depend on $M$ because within the limit of energy resolution, the Zeeman splitting between $M$ levels is not observable, this simplifies further to

$$
\begin{aligned}
S\left(\mathbf{Q}, \mathbf{Q}^{\prime}, E\right)= & 24 \pi \sum_{\mu} \sum_{S} \sum_{m M} Y_{\mu}^{1}(\mathbf{Q} / Q)^{*} Y_{\mu}^{1}\left(\mathbf{Q}^{\prime} / Q^{\prime}\right) \\
& \times\left\langle j_{1}(q)\right\rangle_{E 2 S j}\left\langle j_{1}\left(q^{\prime}\right)\right\rangle_{E 2 S j} \\
& \times\left(\begin{array}{ccc}
1 & 1 & 2 \\
-m & \mu & M
\end{array}\right)^{2} \sum_{j_{z}}(2 j+1) \\
& \times\left(\begin{array}{ccc}
1 & \frac{1}{2} & j \\
m & S & -j_{z}
\end{array}\right) \sum_{\vec{k} n}\left|D_{2 S}^{k n}\right|^{2} \delta\left(E+E_{n l j}-E_{\vec{k} n}\right) .
\end{aligned}
$$

Note that the Bloch wave coefficients $D$ of the final state do not depend on the final magnetic quantum number $M$ any more.

Keeping in mind that we aim at separating transitions with a fixed transfer of angular momentum $(\mu=0, \pm 1)$, we can breakdown expression in Eq. (10)

$$
\begin{aligned}
S^{j}\left(\mathbf{Q}, \mathbf{Q}^{\prime}, E\right)= & 24 \pi \sum_{\mu} Y_{\mu}^{1}(\mathbf{Q} / Q)^{*} Y_{\mu}^{1}\left(\mathbf{Q}^{\prime} / Q^{\prime}\right) \\
& \times\left\langle j_{1}(q)\right\rangle_{E L S j}\left\langle j_{1}\left(q^{\prime}\right)\right\rangle_{E L S j} \\
& \times \sum_{S=\uparrow, \downarrow} C_{j \mu}^{\uparrow, \downarrow} n^{\uparrow, \downarrow},
\end{aligned}
$$

where we have marked the fact that $S$ depends on the relativistic quantum number of the core state $(j=1 / 2$, corresponding to the $L_{2}$ edge, and $j=3 / 2$, corresponding to the $L_{3}$ edge).

The weighting factors $C_{j \mu}^{\uparrow, \downarrow}$ in Eq. (11) are sums over $m, M, j_{z}$,

$$
C_{j \mu}^{\uparrow, \downarrow}=(2 j+1) \sum_{m, M, j_{z}}\left(\begin{array}{ccc}
1 & 1 & 2 \\
-m & \mu & M
\end{array}\right)^{2}\left(\begin{array}{ccc}
1 & \frac{1}{2} & j \\
m & S & -j_{z}
\end{array}\right)^{2},
$$

where $\uparrow, \downarrow$ stand for $S=1 / 2,-1 / 2$. The $\mu$-resolved MDFFs are then weighted sums over spin-up/spin-down population 
TABLE I. Prefactors $C_{j \mu}^{\uparrow, \downarrow}$, Eq. (12). The first two rows are the weighting factors for the transitions when the final states are completely (up or down) spin polarized. The third row gives the weighting factors for unpolarized final states. (All per electron.)

\begin{tabular}{lccccccc}
\hline \hline$j$ & \multicolumn{3}{c}{$1 / 2\left(L_{2}\right)$} & & \multicolumn{3}{c}{$3 / 2\left(L_{3}\right)$} \\
\cline { 2 - 3 } \cline { 6 - 8 }$\mu$ & -1 & 0 & 1 & & -1 & 0 & 1 \\
\hline$\uparrow$ & 0.056 & 0.111 & 0.167 & & 0.278 & 0.222 & 0.167 \\
$\downarrow$ & 0.167 & 0.111 & 0.056 & & 0.167 & 0.222 & 0.278 \\
Unpolarized & 0.111 & 0.111 & 0.111 & & 0.222 & 0.222 & 0.222 \\
\hline \hline
\end{tabular}

densities $n^{\uparrow, \downarrow}=\left|D_{2 S}^{\vec{k} n}\right|^{2}$ of the final state. The sums can be performed analytically. They are given in Table I.

The weighting factors for different $\mu$ are identical when the DOS is unpolarized $\left(n^{\uparrow}=n^{\downarrow}\right)$. Any difference of populations induces deviations of the weighting factors for $\mu$ $= \pm 1$ from that for $\mu=0$. It is also evident from the table that the deviations for the $L_{3}$ edge are opposite to those for the $L_{2}$ edge. Selecting a particular chirality of the transition $(\mu=$ -1 or $\mu=1)$ the transition probabilities will vary in opposite sense for the $L_{2}$ and the $L_{3}$ edges. These variations in the MDFF are directly linked to the dichroic signal measured in EMCD experiments.

In passing we note that the sum over $\mu$ gives

$$
S\left(\mathbf{Q}, \mathbf{Q}^{\prime}\right)=\sum_{L}[l, 1, L]\left\langle j_{1}(q r)\right\rangle\left\langle j_{1}\left(q^{\prime} r\right)\right\rangle\left(\begin{array}{lll}
l & 1 & L \\
0 & 0 & 0
\end{array}\right)^{2} P_{1}(\cos \theta),
$$

where $\theta$ is the angle between $\mathbf{Q}$ and $\mathbf{Q}^{\prime}$ and $P_{1}$ is the Legendre polynomial of order 1 . This expression has been derived earlier for nonmagnetic specimens. ${ }^{33}$

The spin-polarized densities can be calculated from solidstate wave functions (obtained from numerical calculation, e.g., DFT or else). The relative energy shift of the spin-up/ spin-down $d$-projected DOS in Fe causes a spin polarization of almost $100 \%$ in the $L_{23}$ white lines. ${ }^{34}$ We adopt here this approximation and assume total spin polarization of the final $d$ states.

Knowing the spin polarization the energy-filtered HRTEM image of a magnetic transition can be calculated. For a perfect lens the intensity is given as the diagonal element of the density matrix in the exit plane ${ }^{27}$

$$
I(\mathbf{r})=\rho(\mathbf{r}, \mathbf{r}),
$$

where $\rho$, in turn, is the Fourier transform of the density matrix in the diffraction plane,

$$
\rho\left(\mathbf{r}, \mathbf{r}^{\prime}\right)=\mathcal{F}_{\mathbf{q},-\mathbf{q}^{\prime}}\left[\rho\left(\mathbf{q}, \mathbf{q}^{\prime}\right)\right] .
$$

Equations (2), (6), and (11) evaluated at $z=z^{\prime}=0$ yield

$$
I^{j}=\left(\frac{2 \pi m e^{2}}{\varepsilon_{0} \hbar^{2} k}\right)^{2} \mathcal{F}_{\mathbf{q},-\mathbf{q}^{\prime}}\left[S^{j}\left(\mathbf{Q}, \mathbf{Q}^{\prime}\right) / Q^{2} Q^{\prime 2}\right] .
$$

With Eq. (11) we have

$$
I^{j}(\mathbf{r})=\left(\frac{2 \pi m e^{2}}{\varepsilon_{0} \hbar^{2} k}\right)^{2} \sum_{\mu}\left|\psi_{\mu}(\mathbf{r})\right|^{2} \sum_{S=\uparrow, \downarrow} C_{j \mu}^{\uparrow, \downarrow} n^{\uparrow, \downarrow}
$$

with

$$
\psi_{\mu}(\mathbf{r}):=\mathcal{F}_{\mathbf{q}}\left[Y_{\mu}^{1}(\mathbf{Q} / Q)^{*}\left\langle j_{1}(Q)\right\rangle_{E L S j} / Q^{2}\right] .
$$

The Fourier transforms Eq. (15) can be performed, similar to Ref. 27 , by use of a cylindrical coordinate system with the $z$-axis value fixed to $q_{e}$. For $\mathbf{r}$ again in circular coordinates, $\mathbf{r}=(r, \alpha)$ we obtain

$$
\psi_{\mu}(\mathbf{r})=e^{i \mu \alpha} \frac{i^{|\mu|}}{2 \pi} q_{E}^{1-|\mu|} \int_{0}^{\infty} \frac{q^{1+|\mu|} J_{|\mu|}(q r)\left\langle j_{1}(Q)\right\rangle_{E L S j}}{Q^{3}} d q .
$$

This is a convenient result. It tells us that we can construct chiral HR images of systems with spin magnetic moments from three general wave functions $\psi_{\mu}$ and the knowledge of the spin-polarization $n^{\uparrow, \downarrow}$ via the weighting factors. Equation (16) is an extension of an earlier result obtained for nonmagnetic transitions. ${ }^{27}$ The component formerly related to "perpendicular" momentum transfer is now identified as the modulus of $\psi_{ \pm}$, and the "parallel" transfer proportional to $q_{e}$ is identical to $\psi_{0}$ (i.e., designating angular-momentum transfer $\left.L_{z}=0\right)$. The weighting factors $C_{j \mu}$ allow calculations of images for magnetic materials when the final-state spin polarization is known.

It is interesting to see that the handedness of transitions is not visible in the HR image (because the phase of $\psi_{ \pm} 1$ vanishes in the partial intensities). Another detail of Eq. (16) is that the partial intensities $\left|\psi_{\mu}(\mathbf{r})\right|^{2}$ have the same symmetry in the image plane as the projected spherical harmonics $Y_{\mu}^{1}$. This symmetry has been predicted for transitions between eigenstates $|l m\rangle$ and $|l m \pm 1\rangle$, using a different approach in paper I. The present treatment is more general, invoking the Wigner-Eckhart theorem with implicit summation over $m$ states, allowing for spin polarization.

The radial parts of the initial and final wave functions $R_{j S}(r), u_{L S}^{E}(r)$ entering the radial integrals of Eq. (16) can be calculated using different approaches. Results given in the present paper were obtained from wave functions obtained with an all-electron scalar relativistic atomic code based on the DFT. ${ }^{35}$ Calculation was run within the general gradient approximation. Such an atomic approach is expected to give an accurate description of $R_{j S}(r)$ since it is a core state but might be questionable concerning $u_{L S}^{E}(r)$. Undoubtedly, taking the crystal symmetry into account would give better results but involves more complex calculations based on Bloch wave approaches or equivalently, a Green's function formalism. However, due to their high localization on the metallic site, ${ }^{36}$ the $d$ electrons of transition metals are less sensitive to 

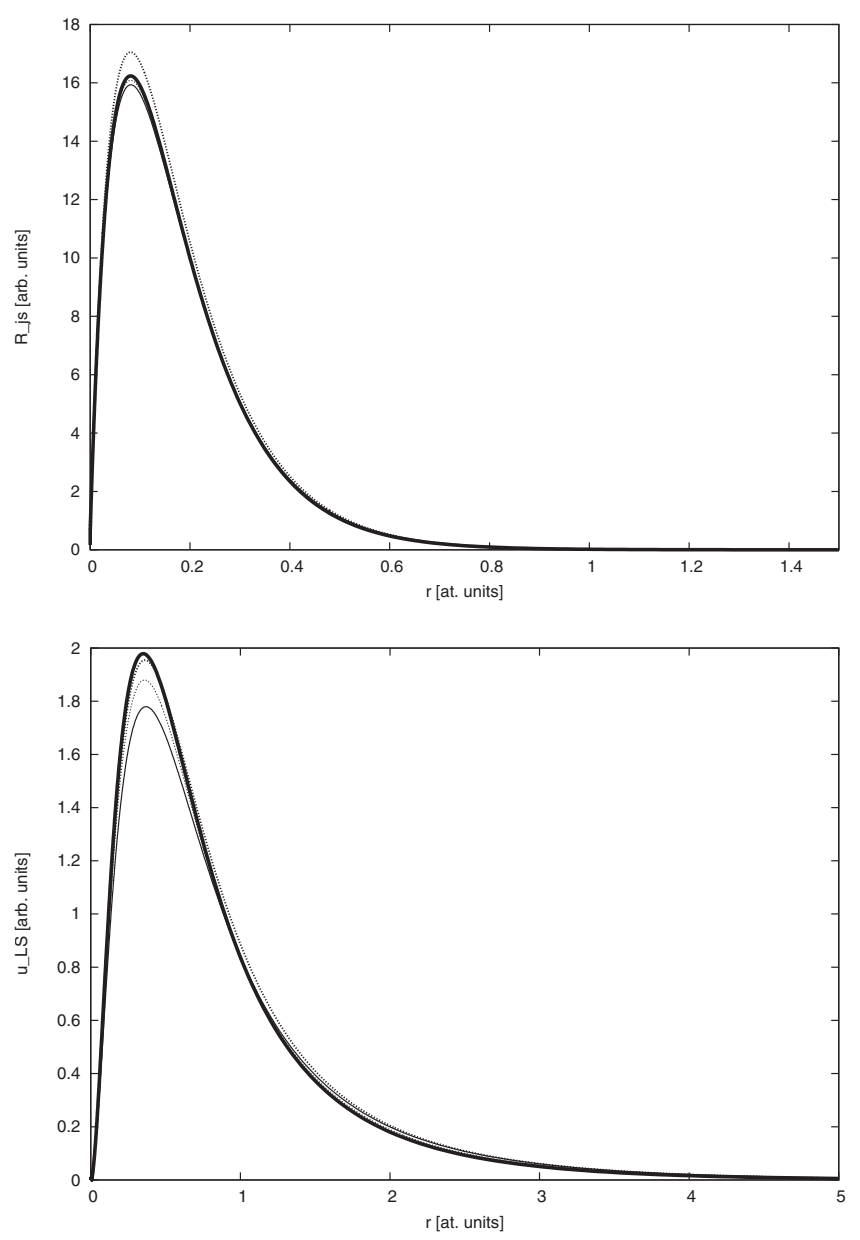

FIG. 1. Radial parts of the iron atomic (a) $2 p$ and (b) $3 d$ wave functions obtained considering a ground-state configuration (thin black lines), the Slater transition state (thin dashed lines), a core hole on a $2 p$ state (bold black lines), and the $Z+1$ approximation (bold dashed lines). Note the different radial range for $2 p$ and $3 d$ states.

solid-state effects than the $s$ or $p$ electrons, and we expect an atomic approach to give a reasonable description of $u_{L S}^{E}(r)$.

When calculating the wave functions involved in electronic transitions, the core-hole created during the excitation process will change the transition probabilities. The influence of such a perturbation on $R_{j S}(r)$ and $u_{L S}^{E}(r)$ was investigated by considering four different approximations: a ground-state approach (corresponding to a $[\mathrm{Ne}] 2 s^{2} 2 p^{6} 3 s^{2} 3 p^{6} 4 s^{2} 3 d^{6}$ configuration), the Slater transition state $\left([\mathrm{Ne}] 2 s^{2} 2 p^{5.5} 3 s^{2} 3 p^{6} 4 s^{2} 3 d^{6.5}\right)$ (Ref. 37), the final-state approximation ([Ne] $\left.2 s^{2} 2 p^{5} 3 s^{2} 3 p^{6} 4 s^{2} 3 d^{7}\right)$, and the $Z+1$ approximation. The latter considers the wave functions for a cobalt atom $(Z+1=27)$. The last two approaches should give rather similar results. ${ }^{38}$ Results obtained for the radial parts of the iron $2 p$ and $3 d$ wave functions are given in Figs. 1(a) and $1(b)$, respectively. It can be seen from the figure that the effect of the core hole is to increase but not to shift the maximum of the radial part of the wave function for both states. This corresponds as expected to a contraction of the corresponding charge density. The maximal relative error between a ground state and an excited configuration calculation

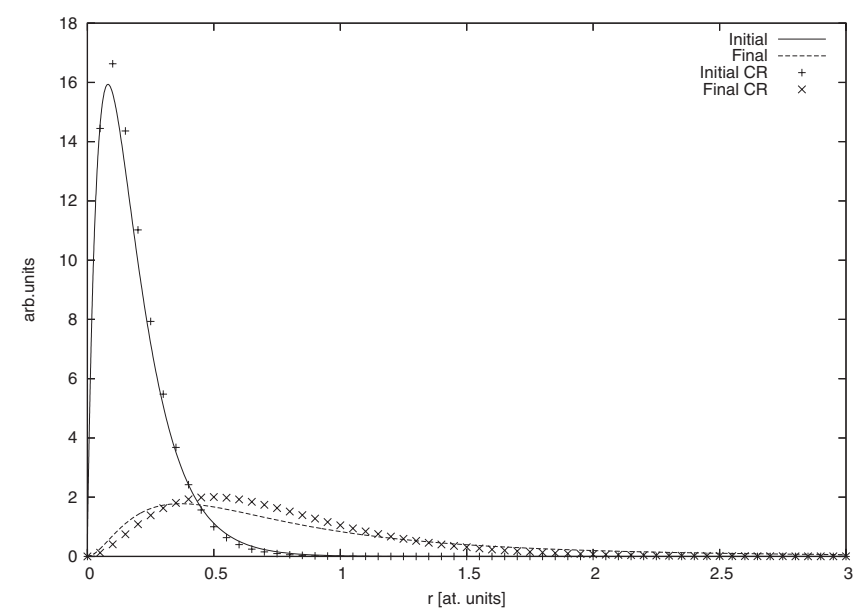

FIG. 2. Radial part of the initial and final all-electron wave functions (calculated with core hole), compared to the ClementiRaimondi approximation (symbols).

is around $7 \%$ for the $2 p$ wave function and $12 \%$ for the $3 d$ one. As illustrated by Fig. 4, these variations do not modify the calculations of the partial intensities within the $q$ range we are interested in. Ground-state or excited state wave functions can be used without any major difference on the resulting image contrast.

Depending on the spin state of the excited electron, the occupation of a given $3 d$ orbital can also be modified. The influence of the occupation on the radial part of the $3 d$ wave function has also been investigated for occupations going from 0 to 2 electrons. The error found is of the same order than that obtained with the core hole. Here again, we expect such a perturbation to have minor consequences on the resulting simulated image contrast.

One can speed-up numerical calculations via Eqs. (14) and (16) considerably by use of an analytical expression for the wave functions. We tried here a simple approach based on Clementi-Raimondi (CR) hydrogenlike atomic wave functions. ${ }^{39}$ They are shown in Fig. 2 together with the allelectron results. The CR $d$-state wave function is narrower than the all-electron one. However, this difference becomes very small when building the product of initial and final states that enters Eq. (8). Figure 3 shows $\left\langle j_{\lambda}(q)\right\rangle_{E L S j}$ for the DFT wave functions with and without core hole, compared to the CR-based wave functions. The differences are small, especially in the $q$ range of interest given by the usual objective apertures $<50 \mathrm{mrad}$. Also shown is the linear approximation (also known as dipole approximation, not to be confounded with the dipole allowed transitions used here- $\left\langle j_{1}(q)\right\rangle_{E L S j}$-which is equivalent to the first term in the Rayleigh expansion of the interaction operator). The linear approximation has a cutoff wave number at the maximum of the Bethe ridge, ${ }^{27} q_{c}=7.88$ a.u. for atomic Fe.

When calculating the density matrix at the exit surface of the specimen all $q$ values must be taken into account, and the Rayleigh expansion must be used. Under realistic conditions, however, with apertures $\beta<50 \mathrm{mrad}$ available in modern objective lenses, the linear approximation is reasonably accurate. 


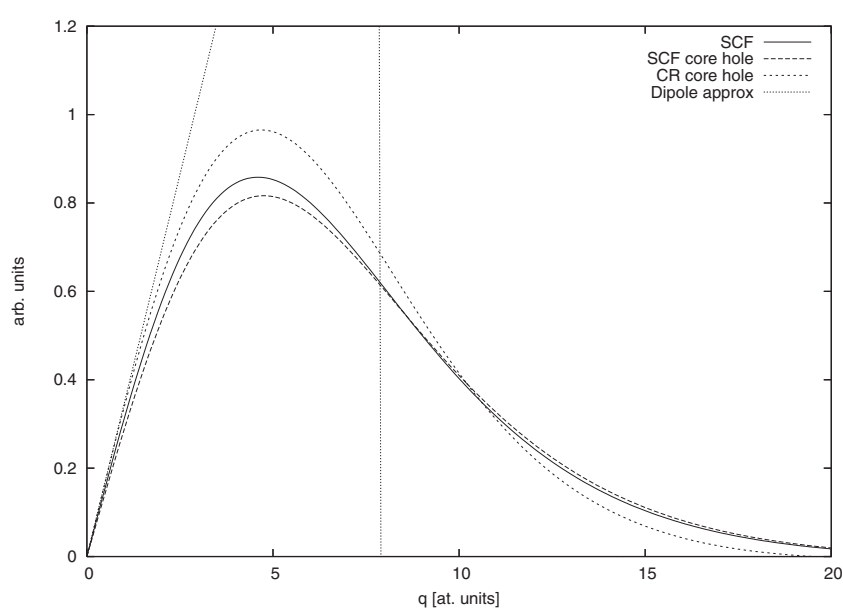

FIG. 3. Radial part $\left\langle j_{\lambda}(q)\right\rangle_{E L S j}$ Eq. (8) for all-electron calculations with (dashed) and without (full line) core hole, and for the CR approximation (dotted). Also shown (and rising beyond the drawing) is the linear (or dipole) approximation with the cutoff wave number at the Bethe ridge.

Figure 4 shows the partial intensities $I_{\mu}$ for a $2 p \rightarrow 3 d$ transition in Fe. For the calculation CR wave functions were used.

The distribution for all three partial intensities is radially symmetric. This is a consequence of the disappearance of the chiral phase $e^{i \Delta m \phi}$ characteristic for those transitions (where $\phi$ is the azimuthal angle with respect to the center of the projected atom) in the image.

An ideal objective lens without limiting aperture would produce a radial intensity profile of a single Fe atom equivalent to the sum of the three traces, as shown in Fig. 5 for a hypothetical aperture of 300 mrad radius in the diffraction plane. It is worth mentioning that the real contrast apertures dramatically change the intensity distribution. These are shown for 60, 40, and $20 \mathrm{mrad}$ in Fig. 5, together with the result if the dipole approximation is assumed, with a cutoff of the angular distribution at the Bethe ridge maximum which is found at $59 \mathrm{mrad}$. It can be seen that with decreas-

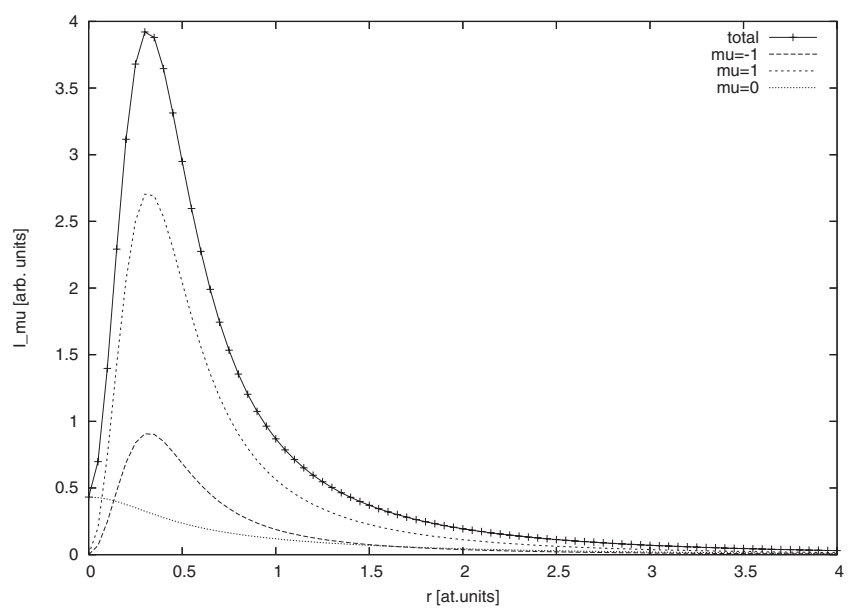

FIG. 4. Radial profiles [partial intensities $I_{\mu}^{1 / 2}(r)$, for $\mu=-1,+1$ (dashed), $\mu=0$ (full line)] and sum profile (symbols) for totally polarized spin $\uparrow$ density.

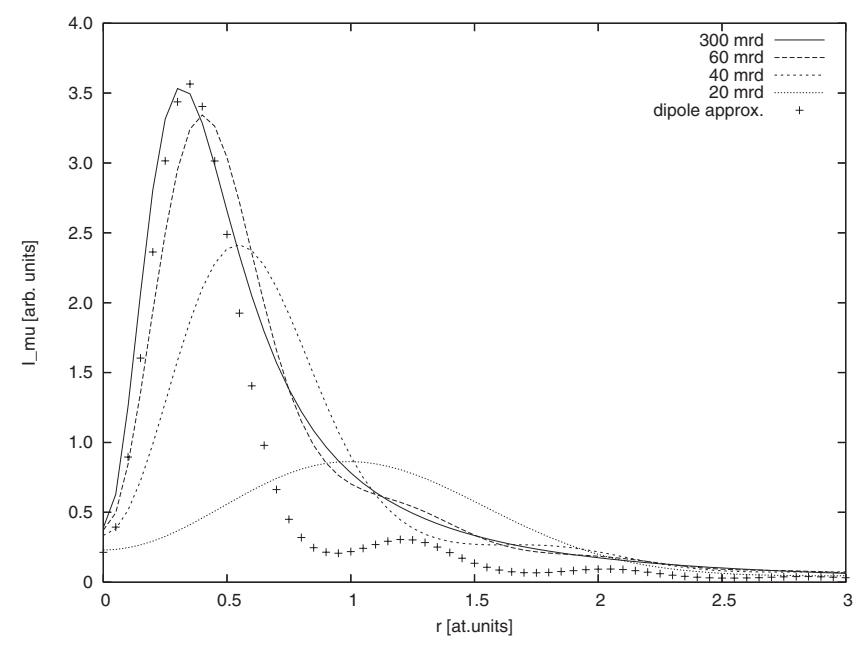

FIG. 5. Radial profiles [sum of partial intensities $I_{\mu}^{1 / 2}(r)$, for $\mu$ $=-1,0,+1]$ for different collection apertures in the diffraction plane. The dipole approximation with a cutoff at the Bethe ridge $\left(q_{c}=7.88\right.$ a.u., corresponding to $59 \mathrm{mrad}$ scattering angle at 200 $\mathrm{kV}$ ) is drawn for comparison (symbols).

ing aperture, the profiles get broader, indicating a reduction in spatial resolution. The dipole approximation has been found to be accurate up to $\approx 30 \mathrm{mrad}$ corresponding to $4 \mathrm{a}$.u. For higher collection angles the deviations become important. This can be understood from Fig. 3. Up to 4 a.u. the dipole approximation reproduces the slope of the CR approximation. The side maxima in the dipole approximation are a consequence of the sharp cutoff by the aperture (or by the Bethe ridge in the present case).

In paper I it was shown that phase information and thus information on the chirality of the transition can be retained by blocking a part of the diffraction plane. The reason is essentially that the azimuthal phase change transforms into a descending/ascending phase ramp in the upper/lower half of the diffraction pattern. After Fourier transform these phase ramps show up as left/right shifts of the intensity profile in the image plane.

The resulting images are shown in Fig. 6. Figure 7 compares images of an $\mathrm{Fe}$ atom with complete spin polarization with that of a hypothetical one without polarization. Also
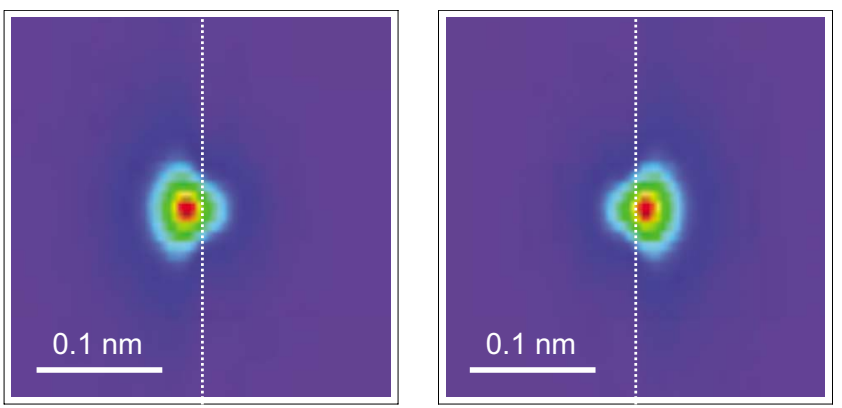

FIG. 6. (Color online) $L_{3}$-edge images of a single Fe atom with the upper/lower half diffraction plane blocked. The asymmetry mirrors the angular momentum of the probe after having caused a chiral electronic transition. The dashed white line marks the center as a guide to the eyes. 

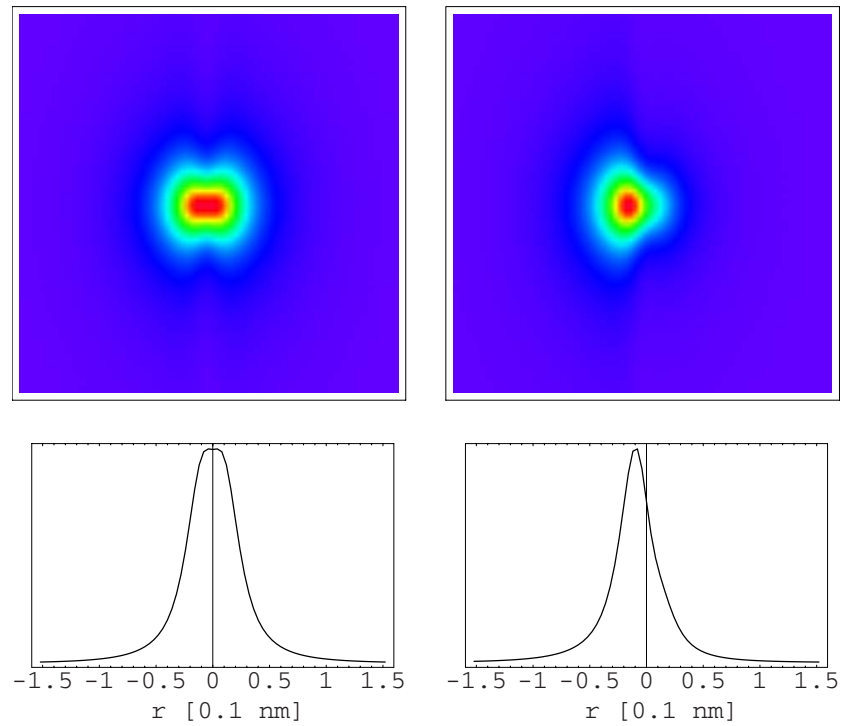

FIG. 7. (Color online) $L_{3}$-edge images of a single Fe atom with the upper half diffraction plane blocked, left: without and right: with spin polarization along the optical axis. The traces are through the center of the atom. Same scale as in Fig. 6.

shown are traces through the center of the atom. The shift of the maximum is on the order of $25 \mathrm{pm}$. A more realistic case is presented in Fig. 8 using a contrast aperture of $20 \mathrm{mrad}$ radius with an offset of $20 \mathrm{mrad}$ in the negative $q_{y}$ direction in the DP.

The shift of the maxima is small, it should, however, be possible to detect it with appropriate data-analysis software; shifts of atom positions as small as $3 \mathrm{pm}$ have been reported $^{40}$ for elastic images. The main problem is the low intensity in this "chiral dark field" (CDF) mode. Subtraction of two CDF images, with positive/negative aperture offset, would double the shift. A trace through such a difference image is shown in Fig. 9, scaled to the intensity maximum. The relative difference is as high as $60 \%$ for the half-plane geometry, higher than the XMCD dichroic signal of Fe.

\section{ELASTIC SCATTERING}

It was shown in paper I that in a crystal, Eq. (1) is replaced by

$$
\begin{aligned}
\rho_{o}(\mathbf{r}, \mathbf{r})= & \iint \bar{P}_{d-z}(\mathbf{r}, \mathbf{x}) \bar{P}_{d-z}^{*}\left(\mathbf{r}^{\prime}, \mathbf{x}^{\prime}\right) T_{z z^{\prime}}\left(\mathbf{x}, \mathbf{x}^{\prime}\right) \\
& \times \bar{\psi}_{z}^{*}(\mathbf{x}) \bar{\psi}_{z^{\prime}}\left(\mathbf{x}^{\prime}\right) d^{2} x d^{2} x^{\prime} e^{i q_{e}\left(z-z^{\prime}\right)} d z d z^{\prime},
\end{aligned}
$$

where we have separated the integrals along the direction of the optical axis $z, z^{\prime}$, and the inelastic kernel $T$ may now depend on $z, z^{\prime}$. The wave functions $\psi$ of the elastically scattered probe electron at depth $z$ are

$$
\bar{\psi}_{z}(\mathbf{x})=\int \bar{P}_{z}\left(\mathbf{x}, \mathbf{r}_{i}\right) \psi_{i}\left(\mathbf{r}_{i}\right) d^{2} r_{i}
$$

and $q_{E}=k_{o}-k_{i}$ is the minimum wave-vector transfer in the inelastic interaction. For energy losses of $<\sim 1 \mathrm{kV}$ as en-
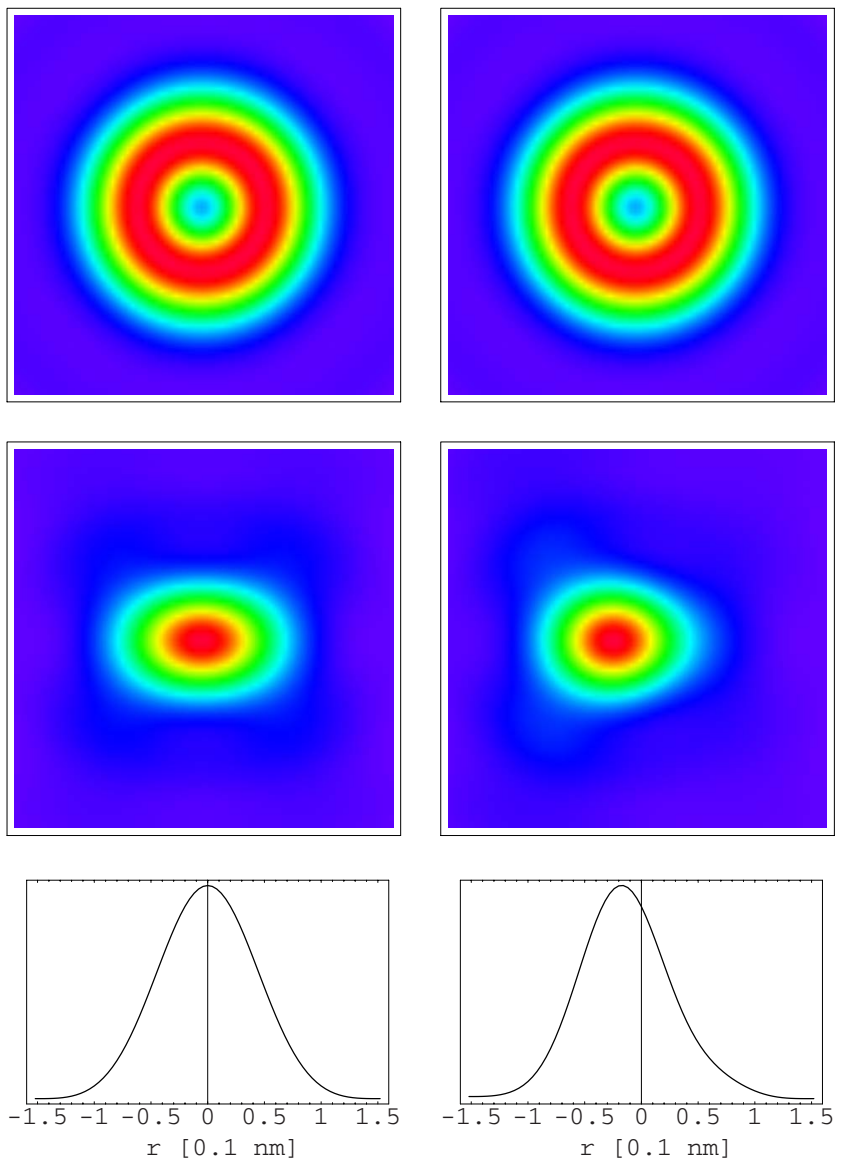

FIG. 8. (Color online) $L_{3}$-edge images of a single $\mathrm{Fe}$ atom. Right column: no spin polarization. Left column: total spin polarization. (a) Upper row: with $20 \mathrm{mrad}$ contrast aperture centered at the optical axis. (b) Middle row: with $20 \mathrm{mrad}$ contrast aperture offset by $20 \mathrm{mrad}$ in the negative $q_{y}$ direction to chiral dark field conditions. (c) Lower row: the profiles are horizontal traces through the center of the atom for case (b) above. Same scale as in Fig. 6.

countered in energy-filtered TEM, the Bloch wave propagators $\bar{P}_{z}$ for the incident and $\bar{P}_{d-z}$ for the inelastically scattered electron can be assumed to be equal which may simplify the calculations. [Note that this is not the case for the propagators $G$ in Eq. (1) which contain an additional phase factor $e^{i k_{f}\left(z-z^{\prime}\right)}$.] We have expressed the $z$ dependence in preparation for the next step.

The wave functions $\bar{\psi}_{z}(\mathbf{x})$ can be calculated with any dynamical scattering code when the incident wave $\psi_{i}\left(\mathbf{r}_{i}\right)$ at the entrance surface $z=0$ is known. The exit plane is at the specimen thickness $z=d$. The density matrix $\rho_{o}$ at the exit plane, Eq. (1), is finally propagated to the detector via $P_{D}$ describing the action of lenses and apertures. At the detector the intensity $I(\mathbf{s})$ is measured (given by the diagonal elements of the density matrix $\rho_{D}$ )

$$
I(\mathbf{s})=\rho_{D}(\mathbf{s}, \mathbf{s})=\int P_{D}(\mathbf{s}, \mathbf{r}) P_{D}^{*}\left(\mathbf{s}, \mathbf{r}^{\prime}\right) \rho_{o}\left(\mathbf{r}, \mathbf{r}^{\prime}\right) d^{2} r d^{2} r^{\prime}
$$

Equations (17)-(19) describe the combination of elastic and inelastic scattering in a crystal. The relevant planes of the 

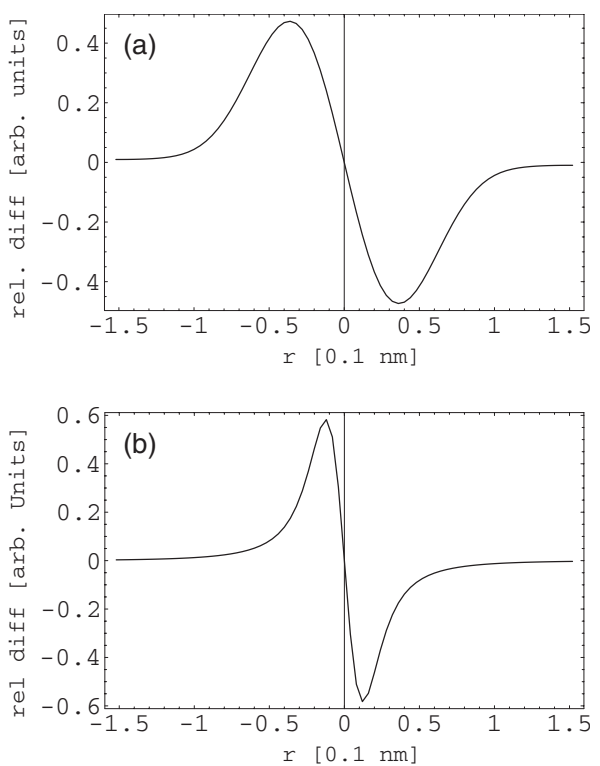

FIG. 9. Horizontal traces through $+/-$ CDF difference maps: $L_{3}$-edge signal of a single $\mathrm{Fe}$ atom (a) with $20 \mathrm{mrad}$ contrast aperture offset by $\pm 20 \mathrm{mrad}$ in the $q_{y}$ direction to dark field conditions. (b) With upper/lower half diffraction plane blocked. The traces are right through the center of the atom. Scaled to the intensity maximum.

experiment are sketched in Fig. 10. Numerically, Eq. (19) poses the following problem: propagation of the incident plane wave via Eq. (18) is straightforward, in particular, when using a multislice approach. The action of the inelastic scattering kernel $T_{z z^{\prime}}$ is also straightforward. The problem arises in the propagation of the inelastically scattered wave further from depth $z$ to the exit surface at $d$. This double channeling effect has been treated recently ${ }^{30,29}$ for nonchiral transitions. We extend the solution and the numerical implementation used in Ref. 29 to chiral transitions, by using the appropriate kernel $T$ derived in the previous section. For a homogeneous crystal and under the locality approximation ${ }^{29}$ (see Appendix) the inelastic scattering kernel does not depend on the depth $z, z^{\prime}$. From Eqs. (2), (14), and (15), we have then

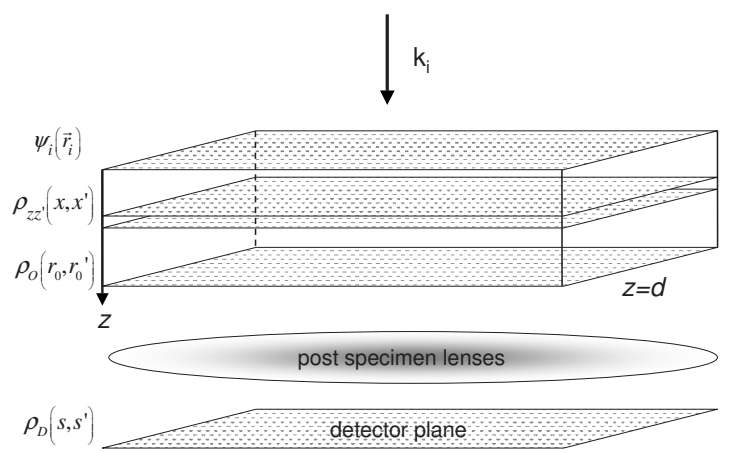

FIG. 10. Position of relevant planes in an inelastic scattering experiment: $\psi_{i}$ at the entrance plane with coordinates $\mathbf{r}_{i}, \rho_{z z^{\prime}}$ at any depth within the specimen, and $\rho_{o}$ at the exit plane. The detector is situated after the postspecimen lens system in the plane $\mathbf{s}$.
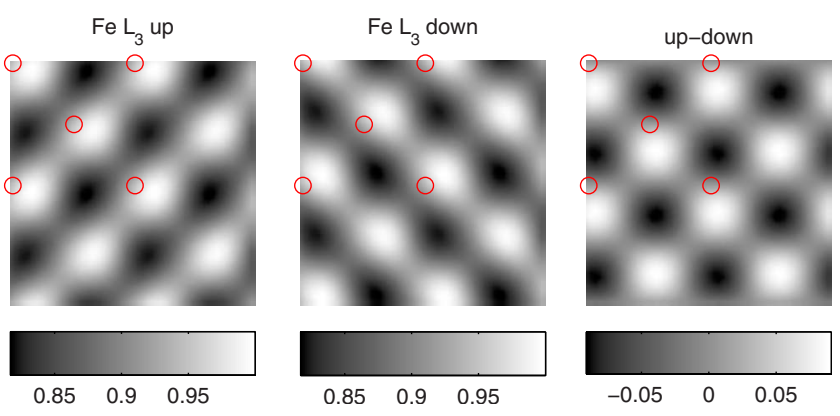

FIG. 11. (Color online) CDF images of $2 \times 2$ elementary cells of an Fe crystal for $10 \mathrm{~nm}$ thickness, Fe $L_{3}$ signal at $200 \mathrm{kV}$. The crystal is oriented in [001] zone axis conditions, aperture $8.8 \mathrm{mrad}$ centered at the (200) Bragg peak. The positions of the Fe atoms are indicated as circles. Left: spin up, middle: spin down. Right: difference map.

$$
T\left(\mathbf{x}, \mathbf{x}^{\prime}\right)=\left(\frac{2 \pi m e^{2}}{\varepsilon_{0} \hbar^{2} k}\right)^{2} \sum_{\mu} \psi_{\mu}(\mathbf{x}) \psi_{\mu}^{*}\left(\mathbf{x}^{\prime}\right) \sum_{S=\uparrow, \downarrow} C_{j \mu}^{\uparrow, \downarrow} n^{\uparrow, \downarrow} .
$$

Each of the three terms in the sum over $\mu$ decompose into products of functions of $\mathbf{x}, \mathbf{x}^{\prime}$, such that the numerics can be simplified by calculating partial wave functions instead of density matrices in the intermediate steps.

Simulations of spin-polarized transitions for a $10-\mathrm{nm}$ thick Fe specimen are shown in Fig. 11. The maps comprise $2 \times 2$ elementary cells with the $\mathrm{Fe}$ positions indicated as red circles. From left to right are shown: spin-up image, spindown image, and the difference map. The intensity wedge shows the contrast in the images. The parameters were as follows: bcc Fe, CR wave functions, $200 \mathrm{kV}$ beam voltage, parallel illumination, crystal in [001]-axis orientation. Collection half angle $8.8 \mathrm{mrad}$, aperture centered at the (200) Bragg position. The pixel size was $0.011025 \mathrm{~nm}$, and a 5 $\times 5$ supercell was used for simulations in order to take the long-range Coulomb coupling into account. A $C_{s}$-corrected objective lens was assumed, and the energy filter was set at the $\mathrm{Fe} L_{3}$ edge.

Whereas atomic-resolution micrographs obtained with elastically scattered electrons would show maxima at the atomic positions (caused by channeling of the probe electrons along the atomic potential wells) the CDF images show a significant asymmetry relative to a horizontal mirror line. The down (up) shift of the maxima is characteristic for the spin-up (spin-down) polarized transitions. These shifts of more than $50 \mathrm{pm}$ are more clearly visible in the difference map to the right. They may be compared with the difference profiles for single atoms, Fig. 9, showing that the elastic scattering on the lattice is not detrimental to the inelastic shift. The image contrast is of order $\pm 10 \%$, much smaller than for the single-atom simulation. This is a consequence of the smaller aperture but also of the elastic scattering that redistributes intensity.

Figure 12 shows the same simulation for a 50 -nm-thick crystal. The individual maps look very different from the 10 $\mathrm{nm}$ case. However, the difference map looks almost identical to the former one. Only the contrast is reduced to $\pm 4 \%$. 

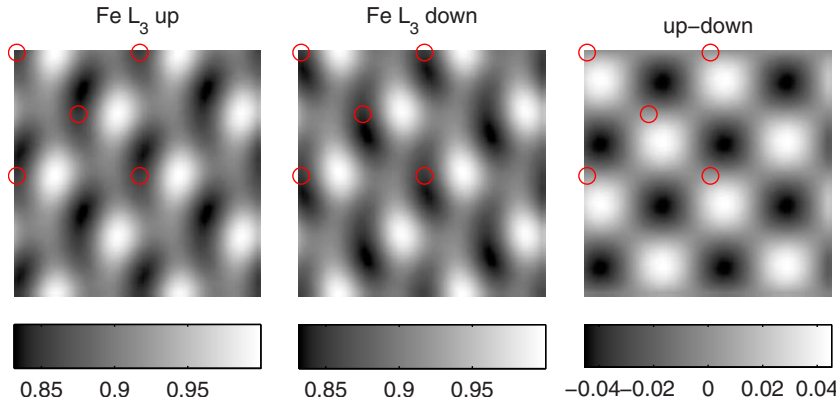

FIG. 12. (Color online) Same as in Fig. 11 for $50 \mathrm{~nm}$ thickness.

These strong variations in the EMCD signal with thickness, with an optimum thickness between 10 and $20 \mathrm{~nm}$ for $\mathrm{Fe}$ at $200 \mathrm{kV}$, were already predicted for diffraction patterns. ${ }^{16}$ The reason is that for thicker crystals the probe electron deposits a considerable amount of angular momentum in the crystal lattice, thus reducing its own chirality and the image asymmetry in CDF conditions.

The problem of correctly disentangling the spindependent asymmetries from the thickness-dependent ones can be solved by subtracting the CDF image from its mirror symmetric one [i.e., centering the aperture at the $(\overline{2} 00)$ Bragg spot]. It can be shown that this results in the same difference images shown in Figs. 9 and 12. ${ }^{41,42}$ The horizontal displacement of the maxima from the atomic positions in the difference spectra is caused by the off-axis position of the objective aperture and is independent of the spin polarization. The vertical displacement is spin sensitive but almost independent of thickness. It is rather the contrast that is controlled by the specimen thickness which is therefore an important optimization parameter. Simultaneous recording of the elastic signal with an annular detector defines a symmetric precise reference frame.

\section{CONCLUSION}

We have outlined the theory of image formation by spinpolarized electronic transitions in a crystal. Numerical simulations for bcc $\mathrm{Fe}$ show that the spin polarization becomes visible on the atomic scale as a significant shift of the maxima in the energy-filtered images. The weakening of the effect in thicker specimens, predicted for diffraction, could be verified in the present atomic imaging configuration. The effect is strong enough to be detectable in ferromagnetic specimens of some $10 \mathrm{~nm}$ thickness. The DFT-based transition probabilities are very close to simpler ones such as CR single-atom models. This fact may simplify predictive simulations which are essential for a thorough understanding of experimental results. In view of the possibility to use electron vortex beams with much higher sensitivity for spinpolarized transitions ${ }^{43}$ the present simulations hold strong promise for experimental detection of spin moments of single atomic columns in electron microscopy.

\section{ACKNOWLEDGMENT}

P.S. acknowledges the support of the Austrian Science Fund, Project No. I543-N20.

\section{APPENDIX}

The locality approximation (the assumption that the inelastic process occurs at a fixed depth $z$ in the specimen or more precisely that we need not consider $z^{\prime} \neq z$ contributions in the propagator) was derived in Ref. 29. For reasons of completeness and because of a changed notation we reproduce it here. The incident fast electron's density matrix $\rho_{i n}$ is propagated elastically as

$$
\rho_{e l}\left(\mathbf{X}, \mathbf{X}^{\prime}\right)=\left\langle\mathbf{X}\left|P_{z}^{+} \rho_{i n} P_{z}\right| \mathbf{X}^{\prime}\right\rangle,
$$

where $P$ is the electron propagator in the crystal. We use coordinates $\mathbf{x}$ perpendicular to the optical axis (in the plane of the specimen) and $z$ along the axis. For ease of demonstration we choose a coherent incident wave with $\rho_{i n}$ $=\left|\phi_{\text {in }}\right\rangle\left\langle\phi_{\text {in }}\right|$. Defining

$$
\left\langle\mathbf{x}\left|P_{z}^{+}\right| \phi\right\rangle:=\phi(\mathbf{X}) e^{i k_{i} z}
$$

we may write

$$
\rho_{e l}\left(\mathbf{X}, \mathbf{X}^{\prime}\right)=\phi(\mathbf{X}) \phi^{*}\left(\mathbf{X}^{\prime}\right) e^{i k_{i}\left(z-z^{\prime}\right)} .
$$

Several comments are in place here. When the incident density matrix describes a pure state then the density matrix at depth $z$ is also a pure state. We obtain it as a product of the propagated incident wave function (this is trivial). $\phi(\mathbf{X})$ is the solution of the Schrödinger equation in the crystal at depth $z$ a superposition of Bloch waves satisfying the entrance boundary conditions). The rapidly varying phase factor $e^{i k_{i}\left(z-z^{\prime}\right)}$ with the incident electron's wave number $k_{i}$ is written explicitly [usually it is ignored since $z=z^{\prime}$ in (elastic) diffraction theory]. The wave function $\phi(\mathbf{X})$ contains still the phase factors $e^{i \gamma^{j} z}$ that make the pendellösung. The propagator $P$ need not carry the depth as an index but we leave it for better recognition in a later stage.

The inelastic scattering kernel $T$ can be shown to act as ${ }^{27}$

$$
\rho_{\text {inel }}\left(\mathbf{X}, \mathbf{X}^{\prime}\right)=T\left(\mathbf{X}, \mathbf{X}^{\prime}\right) \rho_{e l}\left(\mathbf{X}, \mathbf{X}^{\prime}\right) .
$$

This density matrix is now propagated elastically to the detector, according to Eq. (A1)

$$
I_{d e t}(\mathbf{r})=\left\langle\mathbf{r}\left|P_{D}^{+} P_{d-z}^{+} \rho_{\text {inel }} P_{d-z^{\prime}} P_{D}\right| \mathbf{r}\right\rangle .
$$

Inserting unity operators $\int d^{3} X|\mathbf{X}\rangle\langle\mathbf{X}|$ this is

$$
\begin{aligned}
I_{d e t}(\mathbf{r})= & \int \psi_{\mathbf{r}}^{*}(\mathbf{X}) \psi_{\mathbf{r}}\left(\mathbf{X}^{\prime}\right) \\
& \times \phi(\mathbf{X}) \phi^{*}\left(\mathbf{X}^{\prime}\right) e^{i q_{E}\left(z-z^{\prime}\right)} T\left(\mathbf{X}, \mathbf{X}^{\prime}\right) d^{3} X d^{3} X^{\prime},
\end{aligned}
$$

where we have defined the reciprocal wave

$$
\left\langle\mathbf{X}^{\prime}\left|P_{d-z^{\prime}} P_{D}\right| \mathbf{r}\right\rangle:=\psi_{\mathbf{r}}\left(\mathbf{X}^{\prime}\right) e^{i k_{f}\left(d-z^{\prime}\right)} .
$$

The wave function $\psi_{\mathbf{r}}$ is a solution of the Schrödinger equation with boundary conditions given by a point source lo- 
cated at position $\mathbf{r}$ on the detector (this is the reciprocal wave traveling upward the column ${ }^{44}$ ). As such, it is also a superposition of Bloch waves, and we have again extracted a rapidly oscillating phase factor, as before. Note that $k_{i}-k_{f}=q_{E}$, and that the integrals are over the whole specimen volume. Equation (A2) is the kinetic equation. ${ }^{26}$

We have expressed the $z$ dependence in preparation of the next step. The inelastic scattering kernel can be written in configuration space as a convolution of the MDFF with the Coulomb coupling field ${ }^{45}$

$$
T\left(\mathbf{X}, \mathbf{X}^{\prime}\right)=S\left(\mathbf{X}, \mathbf{X}^{\prime}\right)\left(X^{-1} X^{\prime-1}\right) .
$$

The first important step is to assume that the inelastic scattering on different atoms is incoherent. We can then write $S$ as a sum over the different $z$ layers of the crystal

$$
S\left(\mathbf{X}, \mathbf{X}^{\prime}\right)=\sum_{i} S_{a t}\left(\mathbf{x}, z-z_{i}, \mathbf{x}^{\prime}, z^{\prime}-z_{i}\right) .
$$

With this, the rearranged Eq. (A2) reads

$$
I_{d e t}(\mathbf{r})=\int d^{2} x d^{2} x^{\prime} F\left(\mathbf{x}, \mathbf{x}^{\prime}\right)
$$

with

$$
\begin{aligned}
F\left(\mathbf{x}, \mathbf{x}^{\prime}\right)= & \sum_{i} \int d z d z^{\prime} e^{i q_{e}\left(z-z^{\prime}\right)} f(\mathbf{X}) f^{*}\left(\mathbf{X}^{\prime}\right) \\
& \times\left[\left(X^{-1} X^{\prime-1}\right) S_{a t}\left(\mathbf{x}, z-z_{i}, \mathbf{x}^{\prime}, z^{\prime}-z_{i}\right)\right]
\end{aligned}
$$

and

$$
f(\mathbf{X})=\psi_{\mathbf{r}}^{*}(\mathbf{X}) \phi(\mathbf{X}) .
$$

$F$ is a double Fourier transform with respect to $z,-z^{\prime}$. Applying the convolution theorem and the shift theorem we obtain

$$
\begin{aligned}
F\left(\mathbf{x}, \mathbf{x}^{\prime}\right)= & \sum_{i} \mathcal{F}_{z}[f] \mathcal{F}_{-z^{\prime}}\left[f^{*}\right] \\
& \times\left.\left\{K_{0}\left(q_{z} x\right) K_{0}\left(q_{z}^{\prime} x^{\prime}\right) \mathcal{F}_{z,-z^{\prime}}\left[S_{a t}\right] e^{i\left(q_{z}-q_{z}^{\prime}\right) z_{i}}\right\}\right|_{q_{z}=q_{z}^{\prime}=q_{E}} .
\end{aligned}
$$

We have now a closer look on the functions in Eq. (A6). The products of Bloch waves $f$, Fourier transformed with respect to $z$, have deltalike peaks at all combinations of the excitation errors $\gamma^{j}$ of incident and outgoing waves. They are of order $2 \pi / \xi \approx 0.1 \mathrm{rad} \mathrm{nm}^{-1}$. The modified Bessel function $K_{0}$ is smooth in the vicinity of $q_{z}=q_{E}$ where it will be evaluated. The Fourier transform of $S_{a t}$ in dipole approximation is ${ }^{27}$

$$
\begin{aligned}
\mathcal{F}_{z,-z^{\prime}}\left[S_{a t}\right] & =\mathcal{F}_{q,-q^{\prime}}\left[\mathbf{q} \cdot \mathbf{q}^{\prime}+q_{z} q_{z}^{\prime}\right] \\
& =v(\mathbf{x}) v\left(\mathbf{x}^{\prime}\right)+q_{z} q_{z}^{\prime} w(\mathbf{x}) w\left(\mathbf{x}^{\prime}\right), \\
v(\mathbf{x}) & =\frac{1}{2 \pi} \int_{0}^{q_{c}} q^{2} J_{1}(\mathbf{q} \cdot \mathbf{x}) d q, \\
w(\mathbf{x}) & =\frac{q_{z}}{2 \pi} \int_{0}^{q_{c}} q J_{0}(\mathbf{q} \cdot \mathbf{x}) d q,
\end{aligned}
$$

where $q=|\mathbf{q}|$ and $q_{c}$ is the cutoff wave number given by the Bethe ridge. We can then assume that the convolutions with respect to $q_{z}$ of the inelastic kernel with the Bloch waves will shift features by $\delta q_{z} \approx \pm \gamma^{j}$. This has almost no influence at $q_{z} \approx q_{E}$. We can then consider these two functions as constants and extract them from the convolution operation

$$
\begin{aligned}
F\left(\mathbf{x}, \mathbf{x}^{\prime}\right)= & K_{0}\left(q_{z} x\right) K_{0}\left(q_{z}^{\prime} x^{\prime}\right) \mathcal{F}_{z,-z^{\prime}}\left[S_{a t}\right] \\
& \times \sum_{i}\left\{\left.\mathcal{F}_{z}[f] \mathcal{F}_{-z^{\prime}}\left[f^{*}\right] e^{\left.i\left(q_{z}-q_{z}^{\prime}\right) z_{i}\right\}}\right|_{q_{z}=q_{z}^{\prime}=q_{E}} .\right.
\end{aligned}
$$

The convolution here is a Fourier backtransform with an additional phase factor. It yields

$$
\sum_{i} f\left(\mathbf{x}, z_{i}\right) e^{i q_{e} z_{i} f^{*}}\left(\mathbf{x}^{\prime}, z_{i}\right) e^{-i q_{e} z_{i}}
$$

Observing that

$$
K_{0}\left(q_{E} x\right) K_{0}\left(q_{E} x^{\prime}\right) \mathcal{F}_{z,-z^{\prime}}\left[S_{a t}\right]_{q_{E}, q_{E}}=T_{\text {single }}\left(\mathbf{x}, \mathbf{x}^{\prime}\right)
$$

the inelastic kernel for a single layer, we can rewrite Eq. (A9) with Eqs. (A3) and (A10) as

$$
\begin{aligned}
I_{d e t}(\mathbf{r}) \doteq & \sum_{i}\langle\mathbf{r}| P_{D}^{+} P_{d-z_{i}}^{+} T_{\text {single }}\left(\mathbf{x}, \mathbf{x}^{\prime}\right) \\
& \times \phi\left(\mathbf{x}, z_{i}\right) \phi^{*}\left(\mathbf{x}^{\prime}, z_{i}\right) P_{d-z_{i}} P_{D}|\mathbf{r}\rangle .
\end{aligned}
$$

We can sum the intensities from localized planes $z=z_{i}$ incoherently. This is a consequence not only of the incoherence between different atoms but also of the fact that the extinction length of dynamical diffraction is much larger than the extension of the inelastic kernel in $z$ direction.
${ }^{1}$ P. Schattschneider, S. Rubino, C. Hébert, J. Rusz, J. Kuneš, P. Novák, E. Carlino, M. Fabrizioli, G. Panaccione, and G. Rossi, Nature (London) 441, 486 (2006).

${ }^{2}$ F. Houdellier, B. Warot-Fonrose, M. J. Hÿtch, E. Snoeck, L. Calmels, V. Serin and P. Schattschneider, Microsc. Microanal. 13, 048 (2007).

${ }^{3}$ B. Warot-Fonrose, F. Houdellier, M. J. Hÿtch, L. Calmels, V. Serin, and E. Snoeck, Ultramicroscopy 108, 393 (2008).

${ }^{4}$ P. A. van Aken, L. Gu, D. Goll, and G. Schütz, Microsc. Microa- nal. 13, 426 (2007).

${ }^{5}$ H. Lidbaum, J. Rusz, A. Liebig, B. Hjörvarsson, P. M. Oppeneer, E. Coronel, O. Eriksson, and K. Leifer, Microsc. Microanal. 14, 1148 (2008).

${ }^{6}$ Z. H. Zhang, X. Wang, J. B. Xu, S. Muller, C. Ronning, and Q. Li, Nat. Nanotechnol. 4, 523 (2009).

${ }^{7}$ B. Warot-Fonrose, C. Gatel, L. Calmels, V. Serin, E. Snoeck, and S. Cherifi, J. Appl. Phys. 107, 09D301 (2010).

${ }^{8}$ B. Warot-Fonrose, C. Gatel, L. Calmels, V. Serin, and P. 
Schattschneider, Ultramicroscopy 110, 1033 (2010)

${ }^{9}$ H. Lidbaum, J. Rusz, S. Rubino, A. Liebig, B. Hjörvarsson, P. M. Oppeneer, O. Eriksson, and K. Leifer, Ultramicroscopy 110, 1380 (2010).

${ }^{10}$ R. F. Klie, T. Yuan, M. Tanase, G. Yang, and Q. Ramasse, Appl. Phys. Lett. 96, 082510 (2010).

${ }^{11}$ S. Eisebitt, J. Lüning, W. F. Schlotter, M. Lörgen, O. Hellwig, W. Eberhardt, and J. Stöhr, Nature (London) 432, 885 (2004).

${ }^{12}$ D. H. Kim, P. Fischer, W. Chao, E. Anderson, M.-Y. Im, S.-C. Shin, and S.-B. Choe, J. Appl. Phys. 99, 08 H303 (2006).

${ }^{13}$ H. Lichte and M. Lehmann, Rep. Prog. Phys. 71, 016102 (2008).

${ }^{14}$ P. Schattschneider, M. Stöger-Pollach, S. Rubino, M. Sperl, C. Hurm, and J. Zweck, and J. Rusz, Phys. Rev. B 78, 104413 (2008).

${ }^{15}$ L. Calmels, F. Houdellier, B. Warot-Fonrose, C. Gatel, M. J. Hytch, V. Serin, E. Snoeck, and P. Schattschneider, Phys. Rev. B 76, 060409(R) (2007).

${ }^{16}$ J. Rusz, S. Rubino, and P. Schattschneider, Phys. Rev. B 75, 214425 (2007).

${ }^{17}$ H. Lidbaum, J. Rusz, A. Liebig, B. Hjörvarsson, P. M. Oppeneer, E. Coronel, O. Eriksson, and K. Leifer, Phys. Rev. Lett. 102, 037201 (2009).

${ }^{18}$ J. Rusz, H. Lidbaum, A. Liebig, B. Hjörvarsson, P. M. Oppeneer, S. Rubino, O. Eriksson, and K. Leifer, J. Magn. Magn. Mater. 322, 1478 (2010)

${ }^{19}$ P. Schattschneider, S. Rubino, and C. Hébert, Encyclopedia of Materials: Science and Technology (Elsevier, 2007), p. 1.

${ }^{20}$ P. Schattschneider, C. Hébert, S. Rubino, M. Stöger-Pollach, J. Rusz, and P. Novák, Ultramicroscopy 108, 433 (2008).

${ }^{21}$ P. Schattschneider, J. Verbeeck, and A. L. Hamon, Ultramicroscopy 109, 781 (2009).

${ }^{22}$ P. Schattschneider, I. Ennen, M. Stöger-Pollach, J. Verbeeck, V. Mauchamp, and M. Jaouen, Ultramicroscopy 110, 1038 (2010).

${ }^{23}$ I. Grinberg, N. J. Ramer, and A. M. Rappe, Phys. Rev. B 62, 2311 (2000).

${ }^{24}$ L. D. Landau, Z. Phys. 45, 430 (1927).

${ }^{25}$ J. von Neumann, Nachrichten von der Gesellschaft der Wissenschaften zu Göttingen aus dem Jahr 1927 (Weidmannsche Buchhandlung, Berlin, 1928), pp. 273-291.

${ }^{26}$ S. L. Dudarev, L. M. Peng, and M. J. Whelan, Phys. Rev. B 48,
13408 (1993).

${ }^{27}$ P. Schattschneider, M. Nelhiebel, and B. Jouffrey, Phys. Rev. B 59, 10959 (1999).

${ }^{28}$ R. F. Egerton, Electron Energy-Loss Spectroscopy in the Electron Microscope, 2nd ed. (Plenum Press, New York, 1996).

${ }^{29}$ J. Verbeeck, P. Schattschneider, and A. Rosenauer, Ultramicroscopy 109, 350 (2009).

${ }^{30}$ C. Dwyer, S. D. Findlay, and L. J. Allen, Phys. Rev. B 77, 184107 (2008).

${ }^{31}$ F. M. F. de Groot, J. C. Fuggle, B. T. Thole, and G. A. Sawatzky, Phys. Rev. B 42, 5459 (1990).

${ }^{32}$ A. I. Nesvizhskii, A. L. Ankudinov, J. J. Rehr, and K. Baberschke, Phys. Rev. B 62, 15295 (2000).

${ }^{33}$ M. Nelhiebel, Ph.D. thesis, Ecole Centrale Paris, 1999.

${ }^{34}$ M. L. Tiago, Y. Zhou, M. M. G. Alemany, Y. Saad, and J. R. Chelikowsky, Phys. Rev. Lett. 97, 147201 (2006).

${ }^{35}$ R. O. Jones and O. Gunnarsson, Rev. Mod. Phys. 61, 689 (1989).

${ }^{36}$ N. Suaud, G. M. Pastor, S. Evangelisti, and D. Maynau, Chem. Phys. Lett. 378, 503 (2003)

${ }^{37}$ J. C. Slater, The Self-Consistent Field for Molecules and Solids, Quantum Theory of Molecules and Solids (McGraw-Hill, New York, 1974), Vol. 4.

${ }^{38}$ G. Duscher, R. Buczko, S. J. Pennycook, and S. T. Pantelides, Ultramicroscopy 86, 355 (2001).

${ }^{39}$ E. Clementi, D. Raimondi, and W. Reinhardt, J. Chem. Phys. 47, 1300 (1967).

${ }^{40}$ S. Bals, S. Van Aert, G. Van Tendeloo, and D. Ávila Brande, Phys. Rev. Lett. 96, 096106 (2006).

${ }^{41}$ P. Schattschneider, S. Rubino, M. Stoeger-Pollach, C. Hébert, J. Rusz, L. Calmels, and E. Snoeck, J. Appl. Phys. 103, 07D931 (2008).

${ }^{42}$ S. Rubino, P. Schattschneider, M. Stöger-Pollach, C. Hébert, J. Rusz, L. Calmels, B. Warot-Fonrose, F. Houdellier, V. Serin, and P. Novak, J. Mater. Res. 23, 2582 (2008).

${ }^{43}$ J. Verbeeck, H. Tian, and P. Schattschneider, Nature (London) 467, 301 (2010).

${ }^{44}$ Y. Kainuma, Acta Crystallogr. 8, 247 (1955).

${ }^{45}$ J. Verbeeck, G. Bertoni, and P. Schattschneider, Ultramicroscopy 108, 263 (2008) 